\title{
OPEN Mass spectrometric quantitation of AGEs and enzymatic crosslinks in human cancellous bone
}

\author{
Shoutaro Arakawa ${ }^{1,2 \bowtie}$, Ryusuke Suzuki ${ }^{1,2}$, Daisaburo Kurosaka ${ }^{1}$, Ryo Ikeda ${ }^{1}$, \\ Hiroteru Hayashi ${ }^{1}$, Tomohiro Kayama ${ }^{1}$, Rei-ichi Ohno ${ }^{2}$, Ryoji Nagai ${ }^{2}$, Keishi Marumo ${ }^{1}$ \& \\ Mitsuru Saito ${ }^{1}$
}

Advanced glycation end-products (AGEs) deteriorate bone strength. Among over 40 species identified in vivo, AGEs other than pentosidine were roughly estimated as total fluorescent AGEs (tfAGEs) due to technical difficulties. Using LC-OqTOF-MS, we established a system that enabled the quantitation of five AGEs (CML, CEL, MG-H1, CMA and pentosidine) as well as two mature and three immature enzymatic crosslinks. Human bone samples were collected from 149 patients who underwent total knee arthroplasty. Their clinical parameters were collected to investigate parameters that may be predictive of AGE accumulation. All the analytes were quantitated and showed significant linearity with high sensitivity and precision. The results showed that MG-H1 was the most abundant AGE, whereas pentosidine was 1/200-1/20-fold less abundant than the other four AGEs. The AGEs were significantly and strongly correlated with pentosidine, while showing moderate correlation with tfAGEs. Interestingly, multiple linear regression analysis revealed that gender contributed most to the accumulation of all the AGEs, followed by age, tartrate-resistant acid phosphatase-5b and HbA1c. Furthermore, the AGEs were negatively correlated with immature crosslinks. Mass spectrometric quantitation of AGEs and enzymatic crosslinks is crucial to a better understanding of ageing- and disease-related deterioration of bone strength.

Material property of bone is an important determinant of bone strength ${ }^{1}$. The nanoscale structures of bone are formed from collagen fibers surrounded and infiltrated with hydroxyapatite minerals. Collagen fibers provide the material properties such as tensile strength, ductility and toughness, while hydroxyapatite minerals are thought to contribute to stiffness. The functional properties of collagen are influenced by posttranslational modifications (PTMs). Among the modifications, the formation of enzymatic crosslinks between collagen fibrils are essential for physiological bone strength ${ }^{2,3}$. On the contrary, advanced glycation end-products (AGEs) are the results of non-enzymatic PTMs. AGEs are known to form via multiple pathways. In the classical pathway, reducing sugars such as glucose and ribose bind to lysine (Lys) or arginine (Arg) residues in proteins to form Schiff bases and Amadori adducts, which followed by dehydration, oxidation and condensation, eventually leading to the formation of AGEs. The alternative pathways in which reactive carbonyls (i.e. methylglyoxal, glyoxal, glycolaldehyde and glyceraldehyde) bind to Lys or Arg to form AGEs directly are postulated to be the dominant pathway compared to the classical pathway (Fig. 1 $)^{4-6}$. A series of basic and clinical trials have clarified the link between the accumulation of AGEs in bone collagen, and deterioration of bone strength. An in vitro glycation of bovine cortical bone induced pentosidine, an AGE compound, which resulted in reduced stiffness and post-yield strain as evaluated by a 3-point bending test ${ }^{7}$. This phenomenon was also demonstrated in human cancellous bone ${ }^{8}$. An in vivo study involving spontaneously diabetic rats also revealed that after the onset of diabetes, there was an increase in pentosidine accumulation in the femur with decreased bone strength despite no reduction in bone mineral density. Moreover, the link between AGEs and bone strength has been demonstrated in clinical trials. Urinary excretion of pentosidine, which is used as a surrogate marker for bone AGEs, was shown to be a predictor of vertebral fracture after adjustment for age, bone mineral density and renal function ${ }^{9}$. Urinary pentosidine was also shown to improve the accuracy of fracture risk classification, which used the conventional risk assessment tool (Fracture and Immobilization Score: FRISC) ${ }^{10}$. Increasing levels of $N \varepsilon$-(carboxymethyl)

\footnotetext{
${ }^{1}$ Department of Orthopaedic Surgery, Jikei University School of Medicine, 3-25-8, Nishi-Shinbashi, Minato-ku, Tokyo 105-8461, Japan. ' Laboratory of Food and Regulation Biology, School of Agriculture, Tokai University, 9-1-1, Toroku, Higashi-ku, Kumamoto 862-8652, Japan. ${ }^{\circledR}$ email: s.arakawa@jikei.ac.jp
} 


\section{Classical Pathway}

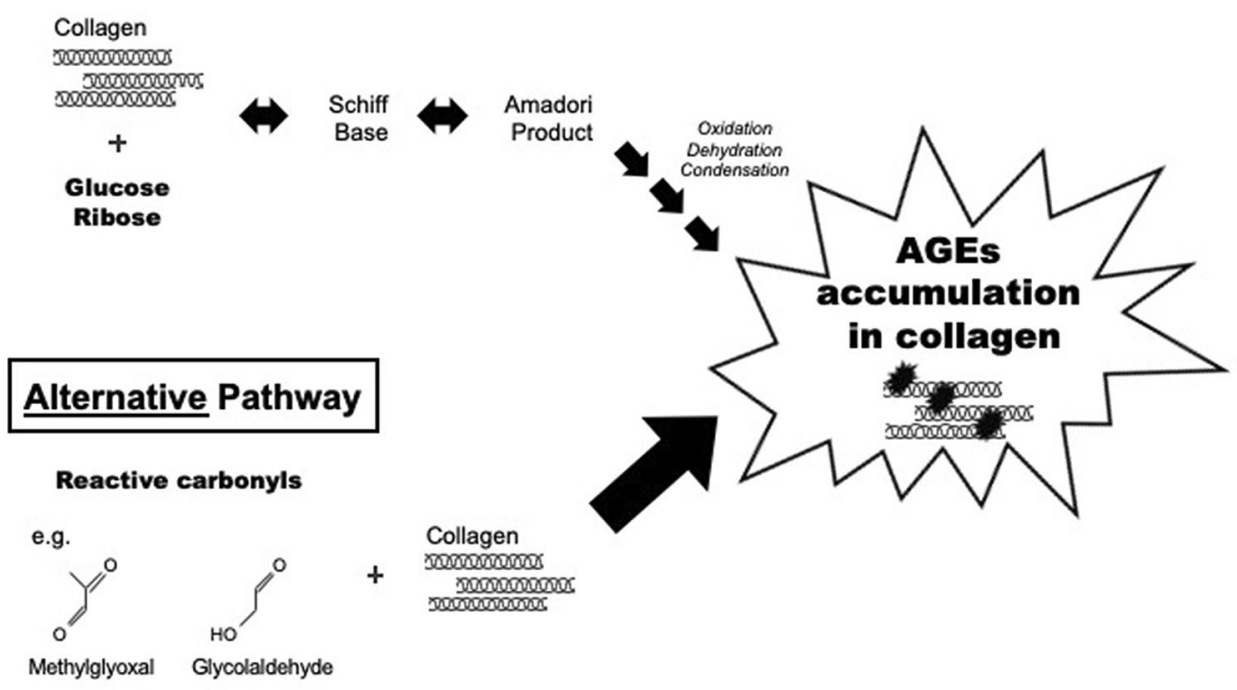

Figure 1. The pathways of AGEs formation.

lysine (CML), another major AGE compound, in serum was also associated with hip fracture risk independent of bone mineral density ${ }^{11}$.

Enzymatic crosslinks have been well-characterized, and five compounds have been quantitated in bone collagen, namely, dihydroxylysinonorleucine (DHLNL), hydroxylysinonorleucine (HLNL), lysinonorleucine (LNL), pyridinoline (PYD) and deoxypyridinoline (DPD $)^{12-14}$. DHLNL, HLNL and LNL are divalent crosslinks between collagen fibrils and are referred to as "immature" crosslinks. Immature crosslinks time-dependently turn into trivalent "mature" crosslinks, PYD and DPD. In contrast, such non-enzymatic modifications as AGEs have not been as thoroughly investigated. Although pentosidine is reported to be associated with the deterioration of bone strength, it is just one out of 40 AGEs that have been identified in vivo ${ }^{2,3,12,15}$. Recently, CML was detected in human bone by ELISA ${ }^{16}$. Other AGEs are estimated using spectrofluorometer and are referred to as total fluorescent AGEs (tfAGEs) ${ }^{17}$. Technological deficits continue to preclude the precise and individual quantitation of different AGEs.

Advancements in analytical technologies have led to the development of mass spectrometry (MS) for the quantitation of various individual glycated adducts. Thornalley et al. ${ }^{18}$, Beisswenger et al. ${ }^{19}$, Schalkwijk et al. ${ }^{20}$, Morcos et al. ${ }^{21}$ and Monnier et al. ${ }^{22}$ have contributed greatly in this field. They analyzed several AGEs and early glycation products in body fluid and soft tissues such as organs and muscles, and sought their potentials as markers or even as causative substances of pathologies such as diabetic complications and chronic kidney disease. We also reported a liquid chromatography (LC)-triple quadrupole MS-based quantitation of CML ${ }^{23}$ and $N^{\delta}$-(5hydro-5-methyl-4-imidazolon-2-yl)-ornithine $1(\mathrm{MG}-\mathrm{H1})^{24}$ in rodent bone. After technological and methodological improvements, we successfully established a system using LC-quadrupole time-of-flight (QqTOF)-MS to quantitate five AGEs and five enzymatic crosslinks in human bone collagen as depicted in Fig. 2. We also quantitated hydroxyproline (Hyp) as a surrogate of collagen content as described previously ${ }^{12}$. Regarding CML formation, it is generated via oxidative degradation of Amadori products, or auto-oxidation of glucose through the production of glyoxal ${ }^{25}$. CML also forms from glycolaldehyde, a reactive carbonyl formed from serine by the action of myeloperoxidase ${ }^{26} \cdot N^{\varepsilon}$-(carboxyethyl)lysine (CEL) is reported to form from the reaction between acetol with $\mathrm{Lys}^{27}$. CEL also forms between Lys and methylglyoxal, another reactive carbonyl generated by degradation of triosephosphate in the glycolytic system (Embden-Meyerhof pathway) ${ }^{28-30}$. MG-H1 forms between Arg and methylglyoxal ${ }^{28}$. $N^{\omega}$-(carboxymethyl)arginine (CMA) forms from Arg and glyoxal, and accumulates specifically in collagen ${ }^{31}$. Pentosidine derives from Lys, Arg and reducing sugars such as glucose and ribose ${ }^{32,33}$. Oxidation of Amadori products is also necessary for the generation of pentosidine ${ }^{27,34}$. An in vitro pathway has also been proposed that pentosidine forms directly from either glycolaldehyde or alternatively glyceraldehyde, a reactive carbonyl formed from oxidative degradation of glucose ${ }^{35}$. As described, the various patterns of AGE formation suggest that each AGE accumulation may differ in vivo, and it is of great importance to quantitate individual AGEs other than pentosidine or tfAGEs. On the other hand, quantitation of enzymatic crosslinks is also necessary as enzymatic crosslinks are determinants of bone strength with a recent report from Hudson et al. suggesting that the formation of enzymatic crosslinks are inhibited by glycation ${ }^{36}$.

In this study, we quantitated five AGEs and five enzymatic crosslinks in 149 human cancellous bone samples by LC-QqTOF-MS to examine the patterns of AGEs accumulation, and to investigate whether pentosidine or tfAGEs more accurately reflects the actual AGEs status in bone collagen. As the clinical manifestations of AGEs accumulation include aging ${ }^{12,37}$, diabetes ${ }^{38}$ and renal failure ${ }^{39}$, we also analyzed the association between AGEs and the clinical parameters such as age, gender, BMI, history of diseases, glycated hemoglobin (HbAlc) as the 


\section{a. CML}

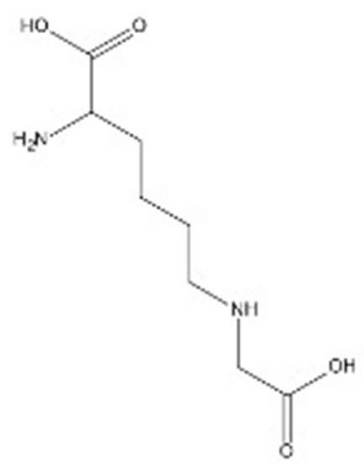

b. CEL

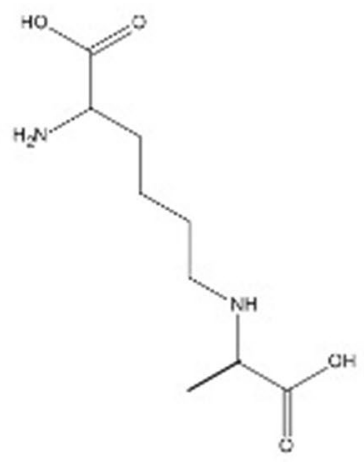

c. MG-H1

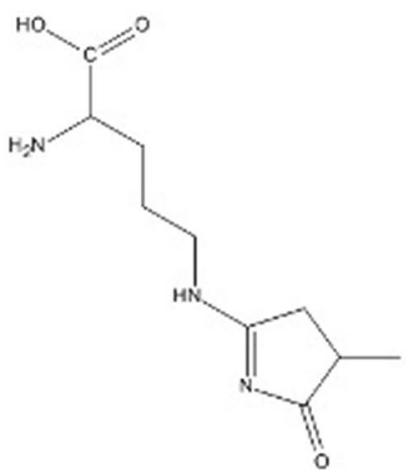

\section{d. CMA}<smiles>N=C(NCCCC(N)C(=O)O)NCC(=O)O</smiles>

\section{e. Pentosidine}<smiles>NC(CCCC[n+]1cccc2nc(NCCCC(N)C(=O)O)[nH]c21)C(=O)O</smiles>

Figure 2. AGEs analyzed in this study. (a) CML, $N^{\varepsilon}$-(carboxymethyl)lysine; (b) CEL, $N^{\varepsilon}$-(carboxyethyl)lysine; (c) MG-H1, $N^{\delta}$-(5-hydro-5-methyl-4-imidazolon-2-yl)-ornithine 1; (d) CMA, $N^{\omega}$-(carboxymethyl)arginine; (e) pentosidine.

\begin{tabular}{|c|c|c|c|c|}
\hline & Total & Male & Female & \\
\hline & $(n=149)$ & $(\mathrm{n}=31)$ & $(n=118)$ & $p$ \\
\hline \multicolumn{5}{|l|}{ Clinical } \\
\hline Age (years) & $73.95 \pm 8.08$ & $74.35 \pm 8.31$ & $73.85 \pm 8.05$ & 0.762 \\
\hline BMI $\left(\mathrm{kg} / \mathrm{m}^{2}\right)$ & $25.72 \pm 3.61$ & $25.34 \pm 2.54$ & $25.81 \pm 3.84$ & 0.435 \\
\hline \multicolumn{5}{|l|}{ History } \\
\hline Hypertension (n, \%) & $78,52.35$ & $13,41.94$ & $65,55.08$ & $\mathrm{n} / \mathrm{a}$ \\
\hline Dyslipidemia (n, \%) & $41,27.52$ & $4,12.90$ & $37,31.36$ & $\mathrm{n} / \mathrm{a}$ \\
\hline \multicolumn{5}{|l|}{ Laboratory } \\
\hline eGFR $\left(\mathrm{mL} / \mathrm{min} / 1.73 \mathrm{~m}^{2}\right)$ & $64.70 \pm 13.01$ & $66.13 \pm 11.33$ & $64.33 \pm 13.44$ & 0.454 \\
\hline HbAlc (\%) & $5.86 \pm 0.41$ & $5.76 \pm 0.43$ & $5.88 \pm 0.40$ & 0.158 \\
\hline TRACP-5b (mU/dL) & $272.0 \pm 127.2$ & $267.3 \pm 107.3$ & $273.2 \pm 132.3$ & 0.797 \\
\hline
\end{tabular}

Table 1. Clinical characteristics of the participants. Values are shown as the mean \pm standard deviation, or as labeled. BMI Body Mass Index, eGFR estimated glomerular filtration rate, TRACP-5b tartrate-resistant acid phosphatase-5b.

marker of blood glycemic status over several weeks to months ${ }^{40}$, tartrate-resistant acid phosphatase-5b (TRACP$5 b)$ as the measure of bone resorption, and estimated glomerular filtration rate (eGFR).

\section{Results}

Clinical characteristics of the patients are summarized in Table 1. There were no gender differences in age, BMI and laboratory data. The prevalence of hypertension and dyslipidemia was higher in females. 
a. CML

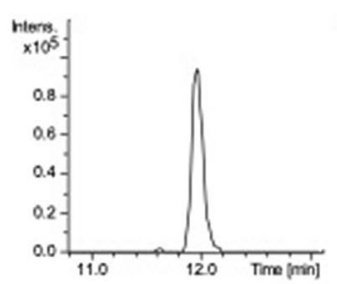

b. CEL

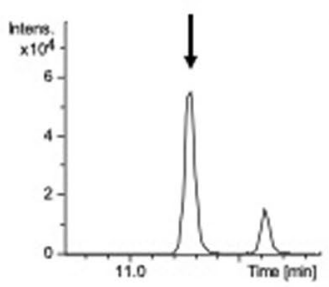

c. MG-H1

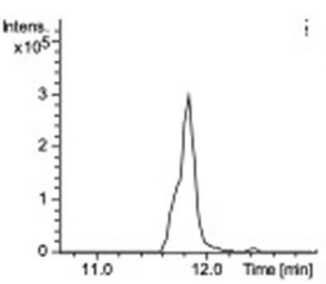

d. CMA

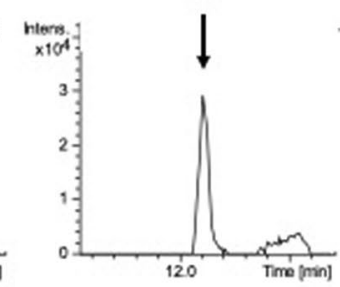

e. Pentosidine

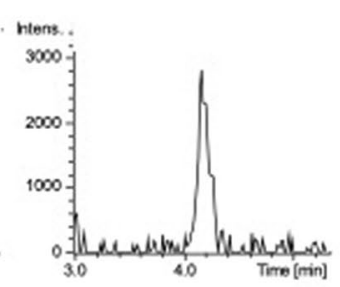

f. DHLNL

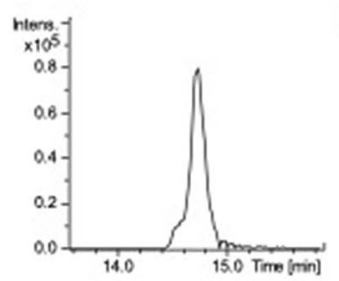

g. HLNL

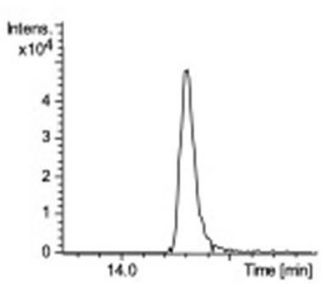

h. LNL

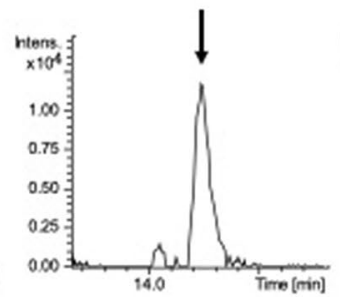

i. PYD

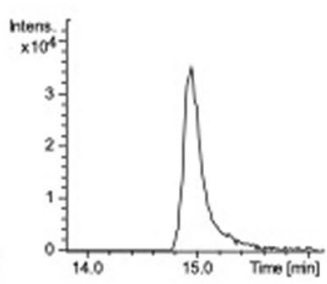

j. DPD

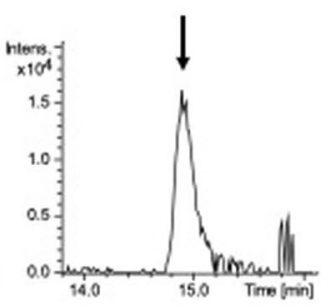

Figure 3. Typical extracted ion chromatographic peaks of AGEs and enzymatic crosslinks (theoretical $\mathrm{m} / \mathrm{z} \pm 0.005)$. (a) CML, $N^{\varepsilon}$-(carboxymethyl)lysine; (b) CEL, $N^{\varepsilon}$-(carboxyethyl)lysine; (c) MG-H1, $N^{\delta}$-(5hydro-5-methyl-4-imidazolon-2-yl)-ornithine 1; (d) CMA, $N^{\omega}$-(carboxymethyl)arginine; (e) pentosidine; (f) DHLNL, dihydroxylysinonorleucine; (g) HLNL, hydroxylysinonorleucine; (h) LNL, lysinonorleucine; (i) PYD, pyridinoline; (j) DPD, deoxypyridinoline.

Typical extracted ion chromatographic peaks of the AGEs and enzymatic crosslinks in human bone hydrolysate are shown in Fig. 3. Linearity, sensitivity, precision and recovery validations are shown in Table 2. All individual AGEs demonstrated linear response when dissolved in $0.1 \%(\mathrm{v} / \mathrm{v})$ formic acid with coefficients of correlation $\left(\mathrm{r}^{2}\right)$ above 0.998 ranging from 0 to $1000 \mathrm{nmol} / \mathrm{L}$. All individual enzymatic crosslinks also demonstrated linear response both in $0.1 \%(\mathrm{v} / \mathrm{v})$ formic acid and in bone hydrolysate with $\mathrm{r}^{2}$ above 0.998 ranging from 0 to $250 \mathrm{nmol} / \mathrm{L}$. Limit of detection (LOD) and limit of quantitation (LOQ) of AGEs were below 14 and $42 \mathrm{nmol} / \mathrm{L}$, respectively. LOD and LOQ of enzymatic crosslinks were below 12 and $35 \mathrm{nmol} / \mathrm{L}$ in $0.1 \%$ formic acid, and below 16 and $48 \mathrm{nmol} / \mathrm{L}$ in bone hydrolysate. Validation of precision showed that intraday coefficients of variations (CVs) were below $5 \%$ for all analytes, whereas interday CVs were slightly greater, especially for enzymatic crosslinks in bone hydrolysate (6-12\%). Recoveries of AGEs were $82-100 \%$ except for MG-H1 (60\%). Recovery rates of enzymatic crosslinks were approximately $50-65 \%$.

MG-H1 was the most abundant AGE among the AGEs quantitated (Fig. 4). Pentosidine was 1/200-1/20-fold less abundant than the other AGEs. Analysis of gender and comorbidities revealed that male gender was associated with statistically higher AGE contents (Supplemental Table S1). The AGEs were higher in hypertensive patients but did not reach statistical significance. There were strong correlations between the AGEs; Spearman's coefficient $\left(r_{s}\right)$ reached 0.9, except for CMA (Table 3). There were moderate correlations between the AGEs and tfAGEs with $r_{s}$ of around 0.3 .

Linear regression analyses were performed to evaluate the relative contributions of clinical parameters to AGE amounts. The result showed that age and HbAlc were positively correlated, while tartrate-resistant acid phosphatase-5b (TRACP-5b) ${ }^{41,42}$, which is an indicator of bone resorption, was negatively correlated with the amount of each AGE (Supplemental Table S2). Subsequent multiple stepwise linear regression analysis, using the amount of each AGE as the dependent variable, with age, body mass index (BMI), estimated glomerular filtration rate (eGFR), TRACP-5b, and gender as independent variables, showed that all parameters except eGFR were independently associated with the amount of each AGE (Table 4). Gender contributed most to the accumulation of all AGEs, followed by age and TRACP-5b. HbA1c was not an independent determinant for pentosidine. Independent contributions of BMI were only significant for CML and MG-H1.

The contents of the enzymatic crosslinks are summarized in Fig. 4. PYD was the most abundant enzymatic crosslink, followed by DHLNL and HLNL. Except for HLNL, there were no differences based on gender (Supplemental Table S3). The maturation index, defined as the ratio of total mature (PYD + DPD) to total immature crosslinks (DHLNL + HLNL $+\mathrm{LNL})$, was positively correlated with age $\left(\mathrm{r}_{\mathrm{s}}=0.271 ; \mathrm{p}<0.001\right)$ and negatively with TRACP-5b $\left(\mathrm{r}_{\mathrm{s}}=-0.237 ; \mathrm{p}<0.01\right)$.

Correlations between each of the lysine-derived AGEs (i.e. CML, CEL and pentosidine), and the enzymatic cross-links were analyzed because these AGEs possibly compete with enzymatic crosslinks for formation sites ${ }^{36}$. The amounts of DHLNL, HLNL, and LNL were negatively correlated with CML, CEL and pentosidine, whereas no such correlation was observed for PYD and DPD (Table 5). 


\begin{tabular}{|c|c|c|c|c|c|c|c|c|c|c|}
\hline \multirow[b]{2}{*}{ Matrix } & \multirow[b]{2}{*}{ Analyte } & \multicolumn{2}{|l|}{ Linearity } & \multicolumn{2}{|c|}{ Sensitivity (nM) } & \multicolumn{2}{|c|}{ Precision (\%) } & \multicolumn{3}{|l|}{ Recovery (\%) } \\
\hline & & Spiked range (nM) & $\mathbf{r}^{2}$ & LOD & LOQ & Intraday & Interday & 10 (pmol) & 20 (pmol) & 40 (pmol) \\
\hline \multirow{14}{*}{$0.1 \%$ formic acid } & \multicolumn{10}{|l|}{ Amino acid } \\
\hline & Hyp & $0-250,000.00$ & 1.000 & 32.43 & 98.27 & 0.50 & 0.63 & - & - & - \\
\hline & AGEs & & & & & & & & & \\
\hline & CML & $0-1000.00$ & 0.998 & 7.74 & 23.45 & 1.72 & 2.19 & - & - & - \\
\hline & CEL & $0-1000.00$ & 1.000 & 13.85 & 41.98 & 1.38 & 1.83 & - & - & - \\
\hline & MG-H1 & $0-1000.00$ & 1.000 & 6.01 & 18.22 & 1.37 & 1.91 & - & - & - \\
\hline & CMA & $0-1000.00$ & 1.000 & 7.69 & 23.30 & 1.95 & 2.42 & - & - & - \\
\hline & Pentosidine & \begin{tabular}{|l|}
$0-500.00$ \\
\end{tabular} & 1.000 & 7.95 & 24.09 & 3.18 & 3.22 & - & - & - \\
\hline & \multicolumn{10}{|c|}{ Enzymatic crosslinks } \\
\hline & DHLNL & $0-1000.00$ & 1.000 & 5.26 & 15.95 & 1.65 & 2.53 & - & - & - \\
\hline & HLNL & $0-1000.00$ & 1.000 & 6.52 & 19.75 & 2.65 & 2.44 & - & - & - \\
\hline & LNL & $0-1000.00$ & 1.000 & 5.61 & 16.99 & 2.06 & 2.02 & - & - & - \\
\hline & PYD & $0-1000.00$ & 1.000 & 11.34 & 34.36 & 4.55 & 6.47 & - & - & - \\
\hline & DPD & $0-1000.00$ & 1.000 & 11.46 & 34.73 & 3.57 & 6.26 & - & - & - \\
\hline \multirow{14}{*}{ Bone hydrolysate } & \multicolumn{10}{|l|}{ Amino acid } \\
\hline & Hyp & $0-250,000.00$ & - & $10,908.52$ & \begin{tabular}{|l|}
$33,056.12$ \\
\end{tabular} & 2.09 & 4.53 & $8.55 \pm 0.11$ & $8.93 \pm 0.03$ & $9.39 \pm 0.04$ \\
\hline & \multicolumn{10}{|l|}{ AGEs } \\
\hline & CML & $0-250$ & 0.997 & 21.20 & 64.23 & 3.37 & 3.46 & $84.64 \pm 2.04$ & $70.71 \pm 0.41$ & $71.35 \pm 1.59$ \\
\hline & CEL & $0-250$ & 0.999 & 13.57 & 41.12 & 4.72 & 3.90 & $92.11 \pm 1.60$ & $86.92 \pm 0.93$ & $85.93 \pm 0.20$ \\
\hline & MG-H1 & $0-1000$ & 0.998 & 71.05 & 215.29 & 3.85 & 3.29 & $72.26 \pm 0.80$ & $64.84 \pm 0.04$ & $70.42 \pm 0.46$ \\
\hline & CMA & $0-250$ & 1.000 & 7.61 & 23.05 & 5.03 & 4.62 & $66.98 \pm 1.05$ & $68.13 \pm 0.38$ & $65.49 \pm 0.98$ \\
\hline & Pentosidine & $0-250$ & 0.999 & 10.14 & 30.72 & 3.62 & 4.94 & $100.48 \pm 7.36$ & $94.30 \pm 2.53$ & $98.08 \pm 2.94$ \\
\hline & \multicolumn{10}{|c|}{ Enzymatic crosslinks } \\
\hline & DHLNL & $0-250$ & 1.000 & \begin{tabular}{|l|}
9.37 \\
\end{tabular} & 28.39 & 2.03 & 12.39 & $58.45 \pm 6.74$ & $58.70 \pm 3.59$ & $52.89 \pm 2.01$ \\
\hline & HLNL & $0-250$ & 1.000 & 10.42 & 31.58 & 4.53 & 6.11 & $55.02 \pm 9.15$ & $54.76 \pm 2.70$ & $51.90 \pm 2.37$ \\
\hline & LNL & $0-250$ & 1.000 & 4.69 & 14.20 & 2.07 & 8.14 & $50.39 \pm 1.14$ & $52.57 \pm 0.99$ & $51.14 \pm 1.01$ \\
\hline & PYD & $0-250$ & 0.999 & 15.65 & 47.41 & 5.43 & 6.35 & $74.89 \pm 5.51$ & $64.07 \pm 2.03$ & $66.54 \pm 2.62$ \\
\hline & DPD & $0-250$ & 1.000 & 10.07 & 30.51 & 3.53 & 7.08 & $47.31 \pm 3.31$ & $53.84 \pm 1.52$ & $52.53 \pm 0.64$ \\
\hline
\end{tabular}

Table 2. Linearity, sensitivity, precision and recovery of the analytes. $L O D$ limit of detection, $L O Q$ limit of quantitation, $H y p$ hydroxyproline, $C M L N^{\varepsilon}$-(carboxymethyl)lysine, $C E L N^{\varepsilon}$-(carboxyethyl)lysine, $M G$ $H 1 N^{\delta}$-(5-hydro-5-methyl-4-imidazolon-2-yl)-ornithine 1, CMA $N^{\omega}$-(carboxymethyl)arginine, DHLNL dihydroxylysinonorleucine, $H L N L$ hydroxylysinonorleucine, $L N L$ lysinonorleucine, $P Y D$ pyridinoline, $D P D$ deoxypyridinoline.

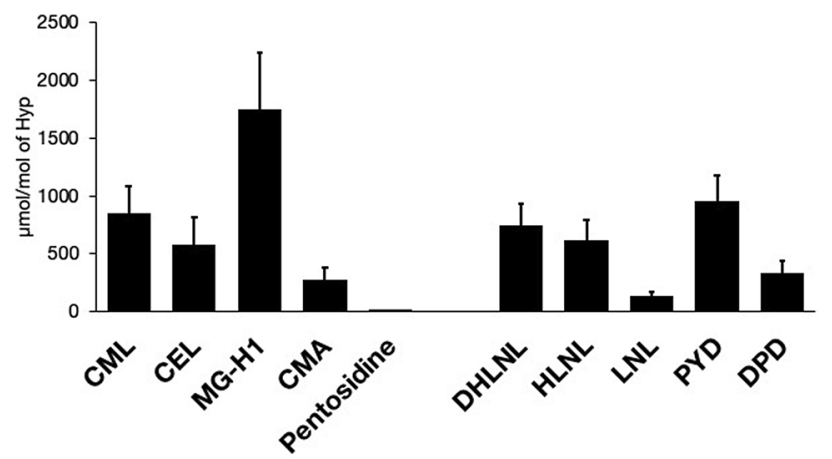

Figure 4. AGEs and enzymatic crosslinks in human cancellous bone from lateral tibia plateau. The filled bar and error bar reflect mean value and standard deviation. Abbreviations: Hyp, hydroxyproline; CML, $N^{\varepsilon}$-(carboxymethyl)lysine; CEL, $N^{\varepsilon}$-(carboxyethyl)lysine; MG-H1, $N^{\delta}$-(5-hydro-5-methyl-4-imidazolon2-yl)-ornithine 1; CMA, $N^{\omega}$-(carboxymethyl)arginine; DHLNL, dihydroxylysinonorleucine; HLNL, hydroxylysinonorleucine; LNL, lysinonorleucine; PYD, pyridinoline; DPD, deoxypyridinoline. 


\begin{tabular}{|l|l|l|l|l|l|}
\hline & CEL & MG-H1 & CMA & Pentosidine & tfAGEs \\
\cline { 2 - 7 } & $\mathbf{r}_{\mathrm{s}}$ & $\mathbf{r}_{\mathrm{s}}$ & $\mathbf{r}_{\mathrm{s}}$ & $\mathbf{r}_{\mathrm{s}}$ & $\mathbf{r}_{\mathrm{s}}$ \\
\hline CML & $0.960^{* * *}$ & $0.933^{* * *}$ & $0.639^{* * *}$ & $0.920^{* * *}$ & $0.400^{* * *}$ \\
\hline CEL & - & $0.922^{* * *}$ & $0.682^{* * *}$ & $0.886^{* *}$ & $0.342^{* * *}$ \\
\hline MG-H1 & - & - & $0.639^{* *}$ & $0.910^{* * *}$ & $0.332^{* * *}$ \\
\hline CMA & - & - & - & $0.614^{* *}$ & 0.002 \\
\hline Pentosidine & - & - & - & - & $0.313^{* *}$ \\
\hline
\end{tabular}

Table 3. Correlation analyses between the AGEs and tfAGEs. $C M L N^{\varepsilon}$-(carboxymethyl)lysine, $C E L$ $N^{\varepsilon}$-(carboxyethyl)lysine, $M G$-H1 $N^{\delta}$-(5-hydro-5-methyl-4-imidazolon-2-yl)-ornithine 1, CMA $N^{\omega}$ (carboxymethyl)arginine, tfAGEs total fluorescent AGEs; $r_{s}$ Spearman's coefficient. ${ }^{\star} \mathrm{p}<0.05 ;{ }^{* *} \mathrm{p}<0.01$; $* * * \mathrm{p}<0.001$.

\begin{tabular}{|l|l|l|l|l|l|}
\hline & CML & CEL & MG-H1 & CMA & Pentosidine \\
\cline { 2 - 6 } & B & B & B & B & B \\
\hline Gender (female) & $-0.424^{* * *}$ & $-0.414^{* * *}$ & $-0.380^{* * *}$ & $-0.232^{* *}$ & $-0.351^{* * *}$ \\
\hline Age & $0.231^{* *}$ & $0.251^{* * *}$ & $0.292^{* * *}$ & $0.194^{*}$ & $0.302^{* * *}$ \\
\hline TRACP-5b & $-0.285^{* * *}$ & $-0.244^{* * *}$ & $-0.254^{* * *}$ & $-0.258^{* * *}$ & $-0.278^{* * *}$ \\
\hline HbAlc & $0.187^{* *}$ & $0.212^{* *}$ & $0.175^{*}$ & $0.179^{*}$ & 0.091 \\
\hline BMI & $0.166^{*}$ & 0.095 & $0.158^{*}$ & - & 0.133 \\
\hline Adjusted $\mathrm{R}^{2}$ & 0.347 & 0.318 & 0.316 & 0.160 & 0.282 \\
\hline
\end{tabular}

Table 4. Multiple stepwise linear regression analysis using each AGE content as the dependent variable, while gender, age, TRACP-5b, HbA1c, BMI and eGFR as the independent variables. CML $N^{\varepsilon}$-(carboxymethyl) lysine, $C E L N^{\varepsilon}$-(carboxyethyl)lysine, $M G-H 1 N^{\delta}$-(5-hydro-5-methyl-4-imidazolon-2-yl)-ornithine 1, $C M A$ $N^{\omega}$-(carboxymethyl)arginine, TRACP-5b, tartrate-resistant acid phosphatase- $5 \mathrm{~b}, \beta$ standardized regression coefficient, BMI Body Mass Index. ${ }^{\star} \mathrm{p}<0.05 ;{ }^{* *} \mathrm{p}<0.01 ;{ }^{* * *} \mathrm{p}<0.001$.

\begin{tabular}{|l|l|l|l|}
\hline \multirow{2}{*}{} & CML & CEL & Pentosidine \\
\cline { 2 - 4 } & $\mathbf{r}_{\mathbf{s}}$ & $\mathbf{r}_{\mathbf{s}}$ & $\mathbf{r}_{\mathbf{s}}$ \\
\hline DHLNL & $-0.417^{* * *}$ & $-0.471^{* * *}$ & $-0.422^{* * *}$ \\
\hline HLNL & $-0.676^{* * *}$ & $-0.695^{* * *}$ & $-0.656^{* * *}$ \\
\hline LNL & $-0.285^{* * *}$ & $-0.350^{* * *}$ & $-0.300^{* * *}$ \\
\hline PYD & 0.010 & -0.004 & -0.011 \\
\hline DPD & 0.026 & -0.037 & 0.000 \\
\hline
\end{tabular}

Table 5. Correlation analyses of the lys-derived AGEs (i.e. CML, CEL and pentosidine) and the enzymatic crosslinks. CML $N^{\varepsilon}$-(carboxymethyl)lysine, CEL $N^{\varepsilon}$-(carboxyethyl)lysine, DHLNL dihydroxylysinonorleucine, $H L N L$ hydroxylysinonorleucine, $L N L$ lysinonorleucine, $P Y D$ pyridinoline, DPD deoxypyridinoline, $r_{s}$ Spearman's coefficient. ${ }^{\star} \mathrm{p}<0.05 ;{ }^{* *} \mathrm{p}<0.01 ;{ }^{* *} \mathrm{p}<0.001$.

\section{Discussion}

In this study, the amounts of CML, CEL, MG-H1, CMA and pentosidine were quantified using LC-QqTOF-MS. Until recently, three methods were applied for the evaluation of AGEs. The first is HPLC equipped with a fluorescence detector (HPLC-FLD) ${ }^{12}$. This method has been reported for the quantitation of pentosidine; however, whether the quantitation of pentosidine is suitable for the elucidation of other AGEs had been unclear. The second method estimated tfAGEs ${ }^{17}$ using a spectrofluorometer. This method cannot detect non-fluorescent AGEs such as CML, and overestimation can occur in the presence of fluorophores other than AGEs. The third method characterizes AGEs using ELISA ${ }^{16}$. Although ELISA is well-characterized and conventional, a cross-reaction can occur and possibly leads to inaccurate analyses especially for AGEs. For example, 6D12, a commercially available anti-CML monoclonal antibody, can cross-react with CEL because CML and CEL are structurally similar ${ }^{43}$. LC-MS can potentially overcome the limitations that HPLC-FLD, spectrofluorometer and ELISA had for two reasons. First, LC-MS detects analytes in their ionized forms, thus does not need analytes to be fluorescent. Second, LC-MS can quantitate analytes with similar structures individually by the combination of retention time and mass-charge ratio $(\mathrm{m} / \mathrm{z})$. Although the five AGEs, whose authentic standards and isotope-labelled internal standards (ISTDs) were commercially accessible, were analyzed in this study, LC-MS will serve to the quantitation of other AGEs in the future. Among them, glucosepane is of great interest because it is thought to be a major crosslinking AGE present in various collagenous tissues ${ }^{22,44}$. 
The developed method was validated for linearity, sensitivity, precision and recovery. All the AGEs and the enzymatic crosslinks demonstrated linear response even beyond physiological ranges. LOD and LOQ of AGEs were comparable with previous studies which utilized triple quadrupole $\mathrm{MS}^{45}$ and quadrupole-Orbitrap $\mathrm{MS}^{46}$. LOD and LOQ of enzymatic crosslinks were also satisfactory compared with the previous study using single quadrupole $\mathrm{MS}^{13}$. Precision validation showed that intraday CVs were below 5\%, but continuous analysis lasting over three days resulted in elevated CVs. This may be due to increased solvent concentration. Therefore in this study, all samples were analyzed within 2 days. Decreased recoveries especially for enzymatic crosslinks were possibly caused by ion suppression ${ }^{47}$. Stable isotope dilution method and standard addition method used in this study are desirable for accurate quantitation using LC-QqTOF-MS ${ }^{47,48}$.

AGEs were analyzed in human bone samples in this study. It was important to take a bone sample undergoing normal remodeling because tissue remodeling is a determinant of AGE accumulation ${ }^{49,50}$. Cadaveric bone is ideal but is not readily available in Japan. Therefore, we took samples from the dissected tibia in total knee arthroplasty. The turnover of cancellous bone in osteoarthritic area might be disturbed, as is the case for subchondral bone ${ }^{51}$. We adopted the sampling method of Oren et al..$^{52}$ and took only the cancellous bone from the central one-third and at a depth of $10 \mathrm{~mm}$ of the lateral tibial plateau where the superficial cartilage was macroscopically intact. Besides the sampling limitation, it was noteworthy that significant correlations were observed here between the AGE contents and age as shown in the previous studies ${ }^{12,16}$.

As described in the introduction, and the alternative pathways that involve reactive carbonyls (i.e. methylglyoxal, glyoxal, glycolaldehyde and glyceraldehyde) are postulated to be the dominant mechanism compared to the classical pathway (Fig. 1) ${ }^{4}$. To support this, the results of this study showed that MG-H1, formed from methylglyoxal and arginine, was the most abundant AGE. On the other hand, pentosidine was much less abundant. MG-H1 content in bone has never been examined previously; however, reports on serum protein ${ }^{18}$, muscle $^{18}$ and Achilles tendons ${ }^{53}$ have shown that MG-H1 accumulation is 200-9,000 times greater than pentosidine accumulation. Methylglyoxal is 20,000 times more reactive than glucose ${ }^{54}$, and this high reactivity could explain the abundance of MG-H1 in various tissues. CML was the second most abundant AGE, accumulating at approximately 100 times the rate of pentosidine. Thomas et al. quantitated CML in human cortical bone using ELISA and compared it with the amount of pentosidine ${ }^{16}$. Although sample type (cancellous versus cortical bone) and analytical methods were different, their results were in accordance with ours in terms of the higher abundance of CML over pentosidine. For a crosslinking AGE to form, two amino acids need to be located at an adequate distance, as shown by an in silico analysis of glucosepane, one of the well-known crosslinking AGEs ${ }^{22,55}$. This stipulation should also apply for pentosidine and thus would have a lower chance of formation when compared with non-crosslinking AGEs such as MG-H1 and CML.

It is technically difficult to quantitate all the individual AGE compounds accumulating in bone collagen, though it is presumed that multiple AGE structures, rather than one specific structure, are involved in the deterioration of bone strength. Thus a surrogate marker such as pentosidine and tfAGEs have been widely used to estimate total AGEs accumulation in bone collagen. However, whether pentosidine or tfAGEs accurately represents other AGEs had not been thoroughly investigated. Thomas et al. found strong correlation $\left(\mathrm{r}^{2}=0.87\right.$, $r<0.05)$ between pentosidine and CML in human cortical bone with limited sample volume $(n=5)^{16}$. Thus in this study, using 149 human cancellous bones, correlations between five AGEs including pentosidine and CML, and tfAGEs were analyzed. As a result, there were strong correlations between the AGEs quantitated in this study, and pentosidine was a better surrogate of other AGEs compared with tfAGEs, at least in human cancellous bone from the tibia. This result was unexpected because AGEs are formed by different pathways ${ }^{4}$ as described earlier, and thus prompted us to perform regression analyses to investigate potential determinants of AGEs accumulation. Surprisingly, in single and multiple regression analyses, gender was the strongest determinant of the AGEs, followed by age, TRACP-5b, HbA1c and BMI. Aging ${ }^{12,38}$, bone turnover ${ }^{49,56}$ and glycative stress ${ }^{38,57}$, which are closely linked, are shown to be determinants for pentosidine accumulation in bone. To the best of our knowledge, there is only one study, reporting gender difference, in which the pentosidine content of human cancellous bone from the femoral head was higher in males ${ }^{58}$. Barp et al. demonstrated that the level of oxidative stress was higher in male rats than in female rats ${ }^{59}$. Data have also been accumulated to show that women are less susceptible to oxidative stress ${ }^{60}$. It was also reported that levels of carbonyl compounds in skin were higher in male turkeys than in females, which raises the possibility that males are more prone to carbonyl stress ${ }^{61}$. The differences of oxidative and carbonyl stress between genders may explain the result that gender was a strong determinant of AGEs accumulation. BMI was a weak but independent determinant of CML and MG-H1. This might be explained by the obesity-related acceleration of oxidative stress seminal to the accumulation of AGEs ${ }^{62}$. Compared with the other AGEs, the adjusted $\mathrm{R}^{2}$ of CMA was less than 0.2 , which implies the presence of other major determinants for CMA that were not examined in this study. It was interesting to find that, unlike other AGEs, HbA1c did not become an independent determinant of pentosidine. HbA1c is a glycated hemoglobin and used as a serological marker of glycemic status over several weeks to months ${ }^{40}$. Although pentosidine formation via the alternative pathway has been implicated in vitro ${ }^{35}$, pentosidine is still presumed to form predominantly through the classical pathway which is a longer process compared to other AGEs pathways ${ }^{4}$. It can be speculated that pentosidine accumulates in bone collagen in association with a longer period of hyperglycemia than required for the accumulation of other AGEs quantitated in this study. Thus HbA1c, which represents hyperglycemic status over only a few months, did not become an independent determinant of pentosidine accumulation. Overall, the strong correlations observed between individual AGE contents indicate that gender, age and bone turnover were strong independent determinants of individual AGEs, irrespective of their pathways. This may be true for the other AGEs not analyzed in this study. From the results of this study, pentosidine is shown to be a more suitable surrogate marker for AGEs accumulation in the human cancellous bone.

The amounts of PYD and DPD detected in this study were consistent with previous reports documenting human cancellous bone in which HPLC-FLD and LC-MS methods were used in conjunction with a conversion 
factor which assumes that collagen weighs 7.5 times the measured weight of Hyp which has a molecular weight of $\sim 300,000$ daltons $s^{12,13}$. The DHLNL amount of human cancellous bone observed by Gineyts et al. ${ }^{13}$ were slightly higher than ours $(0.345 \pm 0.063 \mathrm{vs} 0.231 \pm 0.057 \mathrm{~mol} / \mathrm{mol}$ of collagen). This discrepancy might be attributable to differences in sample types (cancellous tibial bone versus cancellous bone of lumbar vertebrae) and sample extraction methods. The maturation index was positively correlated with age and negatively correlated with TRACP5b. These results are consistent with the findings that immature crosslinks transform into mature crosslinks time-dependently ${ }^{12,13}$.

Interestingly, the results of this study showed that the contents of immature crosslinks were negatively correlated with the contents of Lys-derived AGEs. We previously observed that the contents of femoral pentosidine were elevated in spontaneously diabetic rats whereas immature crosslinks decreased ${ }^{38}$. It was postulated that a hyperglycemia-induced vitamin B6 deficiency caused the down-regulation of lysyl oxidase (LOX) and an eventual reduction in immature crosslinks. Recently, Hudson et al. reported using tail collagen from spontaneously diabetic mice that the sites where immature crosslinks began forming were almost identical to the glycation sites ${ }^{36}$. Thus, the formation of immature crosslinks can be blocked by glycation. In this regard, our findings support the notion of Hudson et al. In terms of abundance, the AGEs other than pentosidine were present to the same order of magnitude as enzymatic crosslinks. Crosslinking AGEs, such as pentosidine and glucosepane, crosslink randomly and can deteriorate the biochemical and mechanical properties of collagen fibrils. Non-crosslinking Lys-derived AGEs, such as CML, on the other hand, can weaken collagen fibrils by blocking the formation of immature crosslinks. With regard to non-crosslinking Arg-derived AGEs such as MG-H1, there has been no study that tried to investigate their effects on bone strength. Given their abundance, future research focusing on their effects on bone strength would be significant.

Several study limitations warrant addressing. First is regarding acid-labile AGEs. In order to determine total AGE contents in proteins using LC-MS, all peptide bonds need to be hydrolyzed. For this, acid hydrolysis in presence of $6 \mathrm{~N} \mathrm{HCl}$ at $110^{\circ} \mathrm{C}$ for $18-24 \mathrm{~h}$ is widely used ${ }^{12,16,52}$. However, acid-labile AGEs, such as glucosepane, break down during this process. Enzymatic hydrolysis is an alternative in this situation, although it is time consuming, expensive, and impractical for routine analysis ${ }^{18}$. Moreover, Antonova, Frolov et al. ${ }^{63}$ pointed out the possibility that compromised solubility of proteins could lead to insufficient enzymatic digestion and result in inaccurate quantitation of AGEs. We opted to add ISTDs before acid hydrolysis so that the losses of partially acid-labile AGEs (i.e. MG-H1 and CMA) during hydrolysis could be corrected. Secondly, the strong correlations between the AGEs in this study may be limited to cancellous bone. Thomas et al. found strong $\left(\mathrm{r}^{2}=0.87, \mathrm{p}<0.05\right.$, $\mathrm{n}=5$ ) correlations between pentosidine and CML in human cortical bone ${ }^{16}$. Meanwhile, Karim et al. found weak correlations $(r=0.226, p<0.05, n=91)$ between pentosidine and tfAGEs in human cortical bone ${ }^{17}$. Factors other than bone turnover are more influential to the accumulation of AGEs because cortical bone turnover is relatively slower $^{64}$. The quantitation of various AGEs in cortical bone is currently in progress. Thirdly, the spatial distribution of AGEs cannot be evaluated using our method of analysis. The efficient use of immunohistochemical techniques may improve the distributional evaluations of AGEs in bone tissue.

In conclusion, mass spectrometric quantitation of AGEs and enzymatic crosslinks in human cancellous bone revealed that pentosidine was 1/200-1/20-fold less abundant than the other AGEs. Unexpectedly, there were strong correlations between the individual AGEs, whereas moderate correlations were observed between the individual AGEs and tfAGEs. Pentosidine, though in itself accumulates in a relatively small quantity, may be a more suitable surrogate marker of other AGEs compared with tfAGEs. In single and multiple regression analyses, gender was the strongest determinant of the AGEs, followed by age, TRACP-5b, HbA1c and BMI. The gender difference in oxidative and carbonyl stress may explain this. In addition, CML and CEL, the non-crosslinking Lys-derived AGEs, were negatively correlated with the immature crosslinks. This result raises the possibility that non-crosslinking AGEs attribute to the deterioration of bone strength by inhibiting the formation of enzymatic crosslinks. Mass spectrometric approach has enabled a detailed analysis of individual AGEs and crosslinks, and is crucial to a better understanding of ageing- and disease-related deterioration of bone strength.

\section{Methods}

Chemical reagents. CML, CEL, MG-H1, $\left[{ }^{2} \mathrm{H}_{2}\right]-\mathrm{CML},\left[{ }^{2} \mathrm{H}_{4}\right]-\mathrm{CEL}$, and $\left[{ }^{2} \mathrm{H}_{3}\right]-\mathrm{MG}-\mathrm{H} 1$ were purchased from PolyPeptide Laboratories (Strasbourg, France). CMA and $\left.{ }^{13} \mathrm{C}_{6}\right]$-CMA were synthesized as described previously ${ }^{31}$. Pentosidine was purchased from Cayman Chemical Company (MI, USA). $\left[{ }^{2} \mathrm{H}_{4}\right]$-Pentosidine was purchased from Alsachim (Illkirch Graffenstaden, France). L-Hyp was purchased from Nacalai Tesque (Kyoto, Japan). $\left[{ }^{2} \mathrm{H}_{3}\right]$-L-Hyp was purchased from C D N Isotopes Inc., (Quebec, Canada). (5S, 5'S)-Dihydroxy lysinonorleucine was purchased from Toronto Research Chemicals (Toronto, Canada). Lysinonorleucine hydrochloride and $\left(2 S, 2^{\prime} S, 5 S\right)$-5-hydroxy lysinonorleucine hydrochloride were purchased from Santa Cruz Biotechnology Inc., (CA, USA). Pyridinoline (PYD) and deoxypyridinoline (DPD) were purchased from TLC Pharmaceutical Standards (Ontario, Canada). High-performance liquid chromatography (HPLC)-grade distilled water $\left(\mathrm{H}_{2} \mathrm{O}\right)$, acetonitrile $(\mathrm{MeCN})$ and formic acid were purchased from Nacalai Tesque (Kyoto, Japan). All other chemicals were purchased from Wako Pure Chemical Inc (Osaka, Japan).

Sample collection. Patients recruited to this study included 149 patients with medial osteoarthritis of the knee who underwent total knee arthroplasty at Jikei University Hospital between 2014 and 2017. Patients with diabetes mellitus, rheumatic and chronic kidney disease on hemodialysis were excluded. Patients taking statins and vitamin D supplementation were also excluded. During surgery, excised tibia samples were immediately frozen and stored at $-80^{\circ} \mathrm{C}$ until biochemical analysis. Blood samples were collected from patients one month prior to surgery and creatinine concentrations and HbAlc were determined subsequently. The estimated glomerular filtration rate (eGFR) was calculated using the following formula: $194 \times$ serum creatinine $\mathrm{s}^{-1.094} \times$ age $^{-0.287}$ 
( $\times 0.739$ if female). Serum TRACP-5b was quantitated using an ELISA kit from DS Pharma Biomedical (Osaka, Japan $)^{65}$ as an indicator of bone resorption ${ }^{66}$. The baseline height and weight of patients was measured for the calculation of BMI.

This study was conducted according to the Declaration of Helsinki and received approval from the Ethics Review Committee of Jikei University Hospital. Each patient provided written informed consent before participating in this study.

Sample preparation. Cancellous bone samples were harvested from the central one-third of the lateral tibia where the superficial cartilages were macroscopically intact and at a depth of $10 \mathrm{~mm}$ from the cartilage surface, and pulverized using a Multi-beads Shocker (Yasui Kikai, Osaka, Japan). Subsequently, bone powders were washed with phosphate-buffered saline $(0.15 \mathrm{~mol} / \mathrm{L} \mathrm{NaCl}$ in sodium phosphate buffer, $\mathrm{pH}$ 7.4), delipidated with chloroform/methanol $(2: 1, \mathrm{v} / \mathrm{v})$ mixture during $24 \mathrm{~h}$ at $4{ }^{\circ} \mathrm{C}$ and demineralized with $0.5 \mathrm{~mol} / \mathrm{L}$ EDTA in $50 \mathrm{mmol} / \mathrm{L}$ Tris buffer ( $\mathrm{pH} 7.4)$ for $96 \mathrm{~h}$ at $4{ }^{\circ} \mathrm{C}$.

Delipidated and demineralized bone powders were reduced by sodium borohydride in sodium borate buffer $(100 \mathrm{mmol} / \mathrm{L}$ of boric acid and $1 \mathrm{mmol} / \mathrm{L}$ diethylenetriamine pentaacetic acid; $\mathrm{pH} 9.1)$ for $4 \mathrm{~h}$ at ambient temperature. Isotope-labelled internal standards (ISTDs) were spiked to reduced bone powders in the following quantities: $5 \mathrm{nmol}$ of $\left[{ }^{2} \mathrm{H}_{3}\right]-\mathrm{L}-\mathrm{Hyp}, 10 \mathrm{pmol}$ of $\left[{ }^{2} \mathrm{H}_{2}\right]-\mathrm{CML},\left[{ }^{2} \mathrm{H}_{4}\right]$-CEL, $\left[{ }^{2} \mathrm{H}_{3}\right]-\mathrm{MG}-\mathrm{H} 1$, and $\left[{ }^{13} \mathrm{C}_{6}\right]-\mathrm{CMA}$, and $20 \mathrm{pmol}$ of $\left[{ }^{2} \mathrm{H}_{4}\right]$-pentosidine. The same quantities of ISTDs were also spiked to six different concentrations of each authentic standard to facilitate the construction of external calibration curves. Both reduced bone powders and standards were hydrolyzed in $6 \mathrm{~N}$ hydrochloric acid $(\mathrm{HCl})$ at $110^{\circ} \mathrm{C}$ for $18 \mathrm{~h}$ and dried using centrifugal evaporation under reduced pressure.

Dried bone hydrolysate $(0.1 \mathrm{mg})$ was resuspended in $\mathrm{H}_{2} \mathrm{O}$ and cleaned up by a cation exchange Strata-X-C solid phase extraction (SPE) column by Phenomenex (CA, USA) to quantitate Hyp, CML, CEL, MG-H1, and CMA. The column was washed with $2 \%$ formic acid and eluted with $7 \%$ ammonia. A separate hydrolysate $(5 \mathrm{mg})$ was cleaned up by a Bond Elut-Cellulose SPE from Agilent Technologies (CA, USA) to quantitate pentosidine. The column was washed with $\mathrm{MeCN} /$ acetic acid/ $\mathrm{H}_{2} \mathrm{O}(8: 1: 1, \mathrm{v} / \mathrm{v} / \mathrm{v})$ mixture and eluted with $\mathrm{H}_{2} \mathrm{O}$. The eluate was dried, re-suspended in $200 \mu \mathrm{L}$ of $0.1 \%$ (v/v) formic acid, filtered through a $0.45 \mu \mathrm{m}$ polytetrafluoroethylene (PTFE) membrane filter from Millex LH, Millipore (MA, USA), and $5 \mu \mathrm{L}$ aliquots were injected into the LC-MS.

LC-MS analysis. For analysis of Hyp, CML, CEL, MG-H1, CMA and enzymatic crosslinks, 0.1 mg of hydrolysate was loaded on a SeQuant ZIC-HILIC column $(5 \mu \mathrm{m}, 150 \times 2.1 \mathrm{~mm})$ by Merck Millipore (MA, USA), installed on a UFLC Nexera from Shimadzu (Kyoto, Japan). The separations were performed at a flow rate of 200 $\mu \mathrm{L} / \mathrm{min}$, at $40{ }^{\circ} \mathrm{C}$ in a linear gradient mode, with eluents $\mathrm{A}$ and $\mathrm{B}$ being water and $\mathrm{MeCN}$, both containing $0.1 \%$ (v/v) formic acid. After an isocratic step at $80 \%$ eluent B for $2 \mathrm{~min}$, analytes were separated in the gradient to $10 \%$ eluent B for $14 \mathrm{~min}$. After a second isocratic segment ( $3 \mathrm{~min}$ at $10 \%$ eluent B) for wash, a third isocratic segment ( $3 \mathrm{~min}$ at $80 \%$ eluent B) was run for re-equilibration. For analysis of pentosidine, $5 \mathrm{mg}$ of hydrolysate was loaded on a SunShell RP-AQUA column $(2.6 \mu \mathrm{m}, 150 \times 2.1 \mathrm{~mm})$ by ChromaNik Technologies (Osaka, Japan), installed on a same LC system and with the same eluents. The separation was performed at a flow rate of $200 \mu \mathrm{L} /$ min, at $40{ }^{\circ} \mathrm{C}$ in an isocratic mode. Pentosidine was separated in an isocratic step at $0 \%$ eluent $\mathrm{B}$ for $4 \mathrm{~min}$. After a second isocratic segment ( 6 min at $60 \%$ eluent $\mathrm{B}$ ) for wash, a third isocratic segment ( 3 min at $0 \%$ eluent B) was run for re-equilibration.

Detection was performed using a micrOTOF-QII quadrupole time-of-flight mass spectrometer (QqTOF-MS) by Bruker Daltonics (Bremen, Germany), equipped with an electrospray ionization source. The instrument was operated in positive ion mode, using a $\mathrm{m} / z$ range of 50-1000. Capillary voltage of ion source was set to $4500 \mathrm{~V}$, nebulizer gas flow was $1.6 \mathrm{bar}$, and dry gas flow was $8 \mathrm{~L} / \mathrm{min}$. Dry temperature was set at $200{ }^{\circ} \mathrm{C}$.

Post-run calibration for each sample was performed by injecting $20 \mu \mathrm{L}$ of mass calibrator at the beginning of each run via the 6-port diverter valve. At each calibration, the measured masses of sodium formate cluster ions were compared with theoretical ones $(\mathrm{m} / z$ 90.976645-974.813156) to achieve $5 \mathrm{ppm}$ mass accuracy. Target analytes and their ISTDs are summarized in Supplemental Table S4. The extracted ion chromatograms (theoretical $\mathrm{m} / z \pm 0.005$ ) were constructed for target analytes and ISTDs, and peak area ratios (target/ISTD) were calculated. The amounts of target analytes in samples were determined by comparing peak area ratios with the 6-point calibration curves of the external standards spiked with ISTDs, respectively. For enzymatic crosslinks, the standard addition method was used because the ISTDs of these crosslinks were commercially unavailable ${ }^{48}$. A separate hydrolysate $(0.05 \mathrm{mg})$ and a hydrolysate $(0.05 \mathrm{mg})$ spiked with 10 pmol of LNL, 20 pmol of DHLNL, HLNL and DPD, and 30 pmol of PYD standards were analyzed. The contents of AGEs and enzymatic crosslinks were standardized to Hyp amounts to surrogate collagen amounts ${ }^{12}$ and expressed as $\mu \mathrm{mol} / \mathrm{mol}$ of Hyp.

HyStar 3.2 for HPLC operation was by Shimazu (Kyoto, Japan). otofControl 4.0 for QqTOF-MS operation, Data Analysis 4.3 for data processing, and QuantAnalysis 4.3 for quantitation were by Bruker Daltonics (Bremen, Germany).

Method validation. The LC-QqTOF-MS method was validated for linearity, sensitivity, precision recovery. Linearity of response for individual AGEs was validated in $0.1 \%(\mathrm{v} / \mathrm{v})$ formic acid, while linearity for enzymatic crosslinks was validated both in $0.1 \%(\mathrm{v} / \mathrm{v})$ formic acid and bone hydrolysate because enzymatic crosslinks were quantified using standard addition method. LOD and LOQ as measures of sensitivity were calculated both in $0.1 \%(\mathrm{v} / \mathrm{v})$ formic acid and bone hydrolysate based on the residual standard deviation of a regression line. Results were expressed as nmol/L. To calculate intraday and interday precisions, authentic standards of all the analytes comparable to physiological concentrations spiked in $0.1 \%$ (v/v) formic acid, and a single bone hydrolysate without standard spike were measured 10 times during the same day and further five times each in two consecutive 
days. Results were expressed as coefficient of variation (\% CV). Recoveries were calculated by spiking 10, 20 and $40 \mathrm{pmol}$ of each standard into bone hydrolysate after SPE. Results were expressed as percentage. All experiments were performed in triplicate.

Measurement of tfAGEs. Bone hydrolysate $(0.5 \mathrm{mg})$ without SPE was used to measure tfAGEs using a spectrofluorometer at wavelengths of $370 \mathrm{~nm}$ excitation and $440 \mathrm{~nm}$ emission (RF-5300PC, Shimadzu, Japan) and normalized to a quinine sulfate standard ${ }^{67}$. TfAGEs was expressed as ng quinine/mol of Hyp.

Statistical analysis. All data are expressed as the mean \pm standard deviation (SD). Differences between groups were examined using the Student's $t$-test. $p$ values of $<0.05$ were considered statistically significant. Spearman's correlation coefficient $\left(r_{s}\right)$ was used to determine the strength and direction of associations between variables and simple regression analyses were performed for univariate correlations. Multiple stepwise regression analyses were performed for multivariate analyses. All statistical analyses were performed using SAS University Edition by SAS Institute Inc. (Cary, NC, USA).

\section{Data availability}

The datasets generated during and/or analyzed during the current study are available from the corresponding author on reasonable request.

Received: 8 May 2020; Accepted: 19 October 2020

Published online: 02 November 2020

\section{References}

1. NIH Consensus Development Panel on Osteoporosis Prevention, Diagnosis, and Therapy. osteoporosis prevention, diagnosis, and therapy. JAMA 285, 785-795 (2011).

2. Saito, M. \& Marumo, K. Collagen cross-links as a determinant of bone quality: A possible explanation for bone fragility in aging, osteoporosis, and diabetes mellitus. Osteoporos. Int. 21, 195-214 (2010).

3. Saito, M. \& Marumo, K. Effects of collagen crosslinking on bone material properties in health and disease. Calcif. Tissue Int. 97, 242-261 (2015).

4. Nagai, R. et al. Detection of AGEs as markers for carbohydrate metabolism and protein denaturation. J. Clin. Biochem. Nutr 55, 1-6 (2014).

5. Sell, D. R., Nemet, I., Liang, Z. \& Monnier, V. M. Evidence of glucuronidation of the glycation product LW-1: Tentative structure and implications for the long-term complications of diabetes. Glycoconj. J. 35, 177-190 (2018).

6. Thorpe, S. R. \& Baynes, J. W. C. M. L. A brief history. Int. Congr. Ser. 1245, 91-99 (2002).

7. Willett, T. L., Sutty, S., Gaspar, A., Avery, N. \& Grynpas, M. In vitro non-enzymatic ribation reduces post-yield strain accommodation in cortical bone. Bone 52, 611-622 (2013).

8. Tang, S. Y., Zeenath, U. \& Vashishth, D. Effects of non-enzymatic glycation on cancellous bone fragility. Bone 40, 1144-1151 (2007).

9. Shiraki, M. et al. Nonenzymatic collagen cross-links induced by glycoxidation (pentosidine) predicts vertebral fractures. J. Bone Miner. Metab. 26, 93-100 (2008).

10. Tanaka, S., Kuroda, T., Saito, M. \& Shiraki, M. Urinary pentosidine improves risk classification using fracture risk assessment tools for postmenopausal women. J. Bone Miner. Res. 26, 2778-2784 (2011).

11. Barzilay, J. I. et al. Circulating levels of carboxy-methyl-lysine (CML) are associated with hip fracture risk: The cardiovascular health study. J. Bone Miner. Res. 29, 1061-1066 (2014).

12. Saito, M., Marumo, K., Fujii, K. \& Ishioka, N. Single-column high-performance liquid chromatographic-fluorescence detection of immature, mature, and senescent cross-links of collagen. Anal. Biochem. 253, 26-32 (1997).

13. Gineyts, E., Borel, O., Chapurlat, R. \& Garnero, P. Quantification of immature and mature collagen crosslinks by liquid chromatography-electrospray ionization mass spectrometry in connective tissues. J. Chromatogr. B 878, 1449-1454 (2010).

14. Berteau, J. P. et al. Ratio between mature and immature enzymatic cross-links correlates with post-yield cortical bone behavior: An insight into greenstick fractures of the child fibula. Bone 79, 190-195 (2015).

15. Saito, M., Kida, Y., Kato, S. \& Marumo, K. Diabetes, collagen, and bone quality. Curr. Osteoporos. Rep. 12, 181-188 (2014).

16. Thomas, C. J., Cleland, T. P., Sroga, G. E. \& Vashishth, D. Accumulation of carboxymethyl-lysine (CML) in human cortical bone. Bone 110, 128-133 (2018).

17. Karim, L., Tang, S. Y., Sroga, G. E. \& Vashishth, D. Differences in non-enzymatic glycation and collagen cross-links between human cortical and cancellous bone. Osteoporos. Int. 24, 2441-2447 (2013).

18. Thornalley, P. J. et al. Quantitative screening of advanced glycation endproducts in cellular and extracellular proteins by tandem mass spectrometry. Biochem. J. 375, 581-592 (2003).

19. Beisswenger, P. J. et al. Early progression of diabetic nephropathy correlates with methylglyoxal-derived advanced glycation end products. Diabetes Care 36, 3234-3239 (2013).

20. Teerlink, T., Barto, R., Ten Brink, H. J. \& Schalkwijk, C. G. Measurement of Ne-(carboxymethyl)lysine and N $\varepsilon$-(carboxyethyl) lysine in human plasma protein by stable-isotope-dilution tandem mass spectrometry. Clin. Chem. 50, 1222-1228 (2004).

21. Morcos, M. et al. Glyoxalase-1 prevents mitochondrial protein modification and enhances lifespan in Caenorhabditis elegans. Aging Cell 7, 260-269 (2008).

22. Sell, D. R. et al. Glucosepane is a major protein cross-link of the senescent human extracellular matrix. J. Biol. Chem. 280, 12310-12315 (2005).

23. Shirakawa, J. et al. Salacia chinensis L. extract ameliorates abnormal glucose metabolism and improves the bone strength and accumulation of AGEs in type 1 diabetic rats. Food Funct. 7, 2508-2515 (2016).

24. Aikawa, T. et al. Contribution of methylglyoxal to delayed healing of bone injury in diabetes. Mol. Med. Rep. 16, 403-409 (2017).

25. Nagai, R. et al. Hydroxyl radical mediates Ne-(carboxymethyl)lysine formation from Amadori product. Biochem. Biophys. Res. Commun. 234, 167-172 (1997).

26. Anderson, M. M., Requena, J. R., Crowley, J. R., Thorpe, S. R. \& Heinecke, J. W. The myeloperoxidase system of human phagocytes generates $\mathrm{N}(\varepsilon)$ - (carboxymethyl)lysine on proteins: A mechanism for producing advanced glycation end products at sites of inflammation. J. Clin. Investig. 104, 103-113 (1999).

27. Nagai, R., Nagai, M., Shimasaki, S., Baynes, J. W. \& Fujiwara, Y. Citric acid inhibits development of cataracts, proteinuria and ketosis in streptozotocin (type 1) diabetic rats. Biochem. Biophys. Res. Commun. 393, 118-122 (2010). 
28. Ahmed, N., Argirov, O. K., Minhas, H. S., Cordeiro, C. A. A. \& Thornalley, P. J. Assay of advanced glycation endproducts (AGEs): Surveying AGEs by chromatographic assay with derivatization by 6 -aminoquinolyl-N-hydroxysuccinimidyl-carbamate and application to Ne-carboxymethyl-lysine- and Ne-(1-carboxyethyl)lysine-modified albumin. Biochem. J. 364, 1-14 (2002).

29. Richard, J. P. Kinetic parameters for the elimination reaction catalyzed by triosephosphate isomerase and an estimation of the reaction's physiological significance. Biochemistry 30, 4581-4585 (1991).

30. Ahmed, M. U., Brinkmann Frye, E., Degenhardt, T. P., Thorpe, S. R. \& Baynes, J. W. Ne-(carboxyethyl)lysine, a product of the chemical modification of proteins by methylglyoxal, increases with age in human lens proteins. Biochem. J. 324, 565-570 (1997).

31. Kinoshita, S. et al. $\mathrm{N} \omega$-(carboxymethyl)arginine is one of the dominant advanced glycation end products in glycated collagens and mouse tissues. Oxid. Med. Cell. Longev. 2019, 1-14 (2019).

32. Sell, D. R. \& Monnier, V. M. Structure elucidation of a senescence cross-link from human extracellular matrix. Implication of pentoses in the aging process. J. Biol. Chem. 264, 21597-21602 (1989).

33. Dyer, D. G., Blackledge, J. A., Thorpe, S. R. \& Baynes, J. W. Formation of pentosidine during nonenzymatic browning of proteins by glucose: Identification of glucose and other carbohydrates as possible precursors of pentosidine in vivo. J. Biol. Chem. 266, 11654-11660 (1991)

34. Nakano, M., Kubota, M., Owada, S. \& Nagai, R. The pentosidine concentration in human blood specimens is affected by heating. Amino Acids 44, 1451-1456 (2013).

35. Chellan, P. \& Nagaraj, R. H. Early glycation products produce pentosidine cross-links on native proteins. Novel mechanism of pentosidine formation and propagation of glycation. J. Biol. Chem. 276, 3895-3903 (2001).

36. Hudson, D. M., Archer, M., King, K. B. \& Eyre, D. R. Glycation of type I collagen selectively targets the same helical domain lysine sites as lysyl oxidase-mediated cross-linking. J. Biol. Chem. 293, 15620-15627 (2018).

37. Wang, X., Shen, X., Li, X. \& Agrawal, C. M. Age-related changes in the collagen network and toughness of bone (Bone (2002) 31 (1-7). Bone 32, 107 (2003).

38. Saito, M., Fujii, K., Mori, Y. \& Marumo, K. Role of collagen enzymatic and glycation induced cross-links as a determinant of bone quality in spontaneously diabetic WBN/Kob rats. Osteoporos. Int. 17, 1514-1523 (2006).

39. Mitome, J. et al. Nonenzymatic cross-linking pentosidine increase in bone collagen and are associated with disorders of bone mineralization in dialysis patients. Calcif. Tissue Int. 88, 521-529 (2011).

40. Rohlfing, C. L. et al. Defining the relationship between plasma glucose and HbA1c: Analysis of glucose profiles and HbAlc in the diabetes control and complications trial. Diabetes Care 25, 1-4 (2002).

41. Nenonen, A. et al. Serum TRACP $5 \mathrm{~b}$ is a useful marker for monitoring alendronate treatment: Comparison with other markers of bone turnover. J. Bone Miner. Res. 20, 1804-1812 (2005).

42. Nakamura, Y. et al. Two-year clinical outcome of denosumab treatment alone and in combination with teriparatide in Japanese treatment-naive postmenopausal osteoporotic women. Bone Res. 5, 1-7 (2017).

43. Ikeda, K. et al. $\mathrm{Ne}$-(Carboxymethyl)lysine protein adduct is a major immunological epitope in proteins modified with advanced glycation end products of the Maillard reaction. Biochemistry 35, 8075-8083 (1996).

44. Fan, X. et al. Anaerobic vs aerobic pathways of carbonyl and oxidant stress in human lens and skin during aging and in diabetes: A comparative analysis. Free Radic. Biol. Med. 49, 847-856 (2010).

45. Nomi, Y. et al. Simultaneous quantitation of advanced glycation end products in soy sauce and beer by liquid chromatographytandem mass spectrometry without ion-pair reagents and derivatization. J. Agric. Food Chem. 64, 8397-8405 (2016).

46. Poojary, M. M. et al. Liquid chromatography quadrupole-Orbitrap mass spectrometry for the simultaneous analysis of advanced glycation end products and protein-derived cross-links in food and biological matrices. J. Chromatogr. A 1615, 460767 (2020).

47. Gosetti, F., Mazzucco, E., Zampieri, D. \& Gennaro, M. C. Signal suppression/enhancement in high-performance liquid chromatography tandem mass spectrometry. J. Chromatogr. A 1217, 3929-3937 (2010).

48. Ito, S. \& Tsukada, K. Matrix effect and correction by standard addition in quantitative liquid chromatographic-mass spectrometric analysis of diarrhetic shellfish poisoning toxins. J. Chromatogr. A 943, 39-46 (2002).

49. Verzijl, N. et al. Effect of collagen turnover on the accumulation of advanced glycation end products. J. Biol. Chem. 275, 39027$39031(2000)$

50. Couppé, C. et al. Mechanical properties and collagen cross-linking of the patellar tendon in old and young men. J. Appl. Physiol. 107, 880-886 (2009)

51. Burr, D. B. \& Gallant, M. A. Bone remodelling in osteoarthritis. Nat. Rev. Rheumatol. 8, 665-673 (2012).

52. Oren, T. W., Botolin, S., Williams, A., Bucknell, A. \& King, K. B. Arthroplasty in veterans: Analysis of cartilage, bone, serum, and synovial fluid reveals differences and similarities in osteoarthritis with and without comorbid diabetes. J. Rehabil. Res. Dev. 48, $1195(2012)$

53. Skovgaard, D. et al. An advanced glycation endproduct (AGE)-rich diet promotes accumulation of AGEs in Achilles tendon. Physiol. Rep. 5, 1-7 (2017).

54. Thornalley, P. J. Dicarbonyl intermediates in the Maillard reaction. Ann. N. Y. Acad. Sci. 1043, 111-117 (2005).

55. Gautieri, A., Redaelli, A., Buehler, M. J. \& Vesentini, S. Age- and diabetes-related nonenzymatic crosslinks in collagen fibrils: Candidate amino acids involved in advanced glycation end-products. Matrix Biol. 34, 89-95 (2014).

56. Saito, M., Fujii, K., Soshi, S. \& Tanaka, T. Reductions in degree of mineralization and enzymatic collagen cross-links and increases in glycation-induced pentosidine in the femoral neck cortex in cases of femoral neck fracture. Osteoporos. Int. 17, 986-995 (2006).

57. Silva, M. J. et al. Type 1 diabetes in young rats leads to progressive trabecular bone loss, cessation of cortical bone growth, and diminished whole bone strength and fatigue life. J. Bone Miner. Res. 24, 1618-1627 (2009).

58. Vaculík, J., Braun, M., Dungl, P., Pavelka, K. \& Stepan, J. J. Serum and bone pentosidine in patients with low impact hip fractures and in patients with advanced osteoarthritis. BMC Musculoskelet. Disord. 17, 1-7 (2016).

59. Barp, J. et al. Myocardial antioxidant and oxidative stress changes due to sex hormones. Brazilian J. Med. Biol. Res. 35, 1075-1081 (2002).

60. Kander, M. C., Cui, Y. \& Liu, Z. Gender difference in oxidative stress: A new look at the mechanisms for cardiovascular diseases. J. Cell. Mol. Med. 21, 1024-1032 (2017).

61. Thomas, C., Dimick, P. \& MacNeill, J. Poultry product quality. 4. Levels of carbonyl compounds in fresh, uncooked chicken and Turkey skin. J. Food Sci. 36, 527-531 (1971).

62. Furukawa, S. et al. Increased oxidative stress in obesity and its impact on metabolic syndrome find the latest version: Increased oxidative stress in obesity and its impact on metabolic syndrome. J. Clin. Investig. 114, 1752-1761 (2017).

63. Antonova, K. et al. Analysis of chemically labile glycation adducts in seed proteins: Case study of methylglyoxal-derived hydroimidazolone 1 (MG-H1). Int. J. Mol. Sci. 20, 3659 (2019).

64. Odetti, P. et al. Advanced glycation end products and bone loss during aging. Ann. N. Y. Acad. Sci. 1043, 710-717 (2005).

65. Nishizawa, Y. et al. Evaluation of newly developed kit for measurement of bone-specific tartrate-resistant acid phosphatase in blood (in Japanese). Jpn. J. Med. Pharm. Sci. 54, 709-717 (2005).

66. Halleen, J. M. Tartrate-resistant acid phosphatase 5B is a specific and sensitive marker of bone resorption. Anticancer Res. 23, 1027-1029 (2003).

67. Mashiba, T. et al. Effects of suppressed bone remodeling by minodronic acid and alendronate on bone mass, microdamage accumulation, collagen crosslinks and bone mechanical properties in the lumbar vertebra of ovariectomized cynomolgus monkeys. Bone 97, 184-191 (2017). 


\section{Acknowledgements}

The authors thank Jun-ichi Shirakawa and Takeo Iwamoto for technical assistance. R.N. has patent on the pretreatment of samples for detection of AGEs using LC-MS (Patent \#6002567).

\section{Author contributions}

S.A. and M.S. designed the experiments. S.A., R.S., R.O. and R.N. contributed the development of methodology. M.S., D.K., R.I., H.H., T.K. and K.M. contributed the collection of samples. S.A. and R.S. performed the experiments and analyzed data. M.S., K.M. and R.N. supervised the experiments. S.A. wrote the manuscript. M.S. and R.N. reviewed the manuscript. All authors read and approved the final version of the manuscript.

\section{Competing interests}

The authors declare no competing interests.

\section{Additional information}

Supplementary information is available for this paper at https://doi.org/10.1038/s41598-020-75923-8.

Correspondence and requests for materials should be addressed to S.A.

Reprints and permissions information is available at www.nature.com/reprints.

Publisher's note Springer Nature remains neutral with regard to jurisdictional claims in published maps and institutional affiliations.

(c) (i) Open Access This article is licensed under a Creative Commons Attribution 4.0 International License, which permits use, sharing, adaptation, distribution and reproduction in any medium or format, as long as you give appropriate credit to the original author(s) and the source, provide a link to the Creative Commons licence, and indicate if changes were made. The images or other third party material in this article are included in the article's Creative Commons licence, unless indicated otherwise in a credit line to the material. If material is not included in the article's Creative Commons licence and your intended use is not permitted by statutory regulation or exceeds the permitted use, you will need to obtain permission directly from the copyright holder. To view a copy of this licence, visit http://creativecommons.org/licenses/by/4.0/.

(C) The Author(s) 2020 